\title{
Linx
}

Revue des linguistes de l'université Paris X Nanterre

$44 \mid 2001$

Spécificité et histoire des discours sémiotiques

\section{Sémiotique et sémantique}

\section{Yong-Ho Choi}

\section{(2) OpenEdition \\ Journals}

Édition électronique

URL : http://journals.openedition.org/linx/1035

DOI : 10.4000/linx.1035

ISSN : 2118-9692

\section{Éditeur}

Presses universitaires de Paris Nanterre

\section{Édition imprimée}

Date de publication : 1 juin 2001

Pagination : 75-84

ISSN : 0246-8743

\section{Référence électronique}

Yong-Ho Choi, « Sémiotique et sémantique », Linx [En ligne], 44 | 2001, mis en ligne le 05 juillet 2012, consulté le 30 avril 2019. URL : http://journals.openedition.org/linx/1035; DOI : 10.4000/linx.1035

Ce document a été généré automatiquement le 30 avril 2019

Département de Sciences du langage, Université Paris Ouest 


\title{
Sémiotique et sémantique
}

\author{
Yong-Ho Choi
}

\section{Introduction}

1 Comment définir la relation entre sémiotique et sémantique ? Cette question reste encore sans réponse définitive. Ainsi s'exprime F. Rastier: "Semantics and semiotics have entertained dubious relationships $»^{1}$. Dans la présente étude, il ne s'agira pourtant pas de préparer à une rencontre: dès l'origine, les deux disciplines se dissocient peu l'une de l'autre, sauf peut-être par leur statut épistémologique respectif. La sémiotique comme la sémantique ont pour préoccupation majeure la quête $d u$ sens $^{2}$. Reste seulement à savoir de quel sens il est question.

2 On ne cherchera pas non plus à ériger une hiérachisation utilitariste aboutissant à la tripartition de la sémiotique en sémantique, syntaxe et pragmatique. Si la sémiotique dépasse la sémantique, elle ne la remplace pas. Elles seraient plutôt en relation de présupposition réciproque. Mon idée est que le sémantique est dans la sémiotique et que le sémiotique est dans la sémantique.

3 Ceci étant dit, mon article s'articulera comme suit : i) le sémantique dans la sémiotique ii) le sémiotique dans la sémantique. Avant d'entrer dans le cœur du sujet, qu'on me permette de tracer les grandes lignes épistémologiques suivant lesquelles mon travail se situera.

\section{Mise au point épistémologique}

Pour la délimitation du champ d'investigation ainsi ouvert, j'emprunterai une voie épistémologique comparative. J'éviterai ainsi de prendre un long détour historique. Pour la mise au point épistémologique, j'aimerais recourir à la triade de Peirce, reprise par Wolfang Widgen sous la forme du Postulat de Peirce: «L'unité fondamentale du signe est donnée par une triade A, B, C et les relations entre les éléments de cette triade : (A, B), (B, 
C) et $(\mathrm{A}, \mathrm{C})$ » $(1994: 164)$. Widgen nous invite ainsi à la reformulation de la triade de Peirce (ibid.) :

- la forme du signe (le « representamen » selon Peirce) est normalement un type de processus (de production verbale ou d'écriture) et son résultat, qui n'est stable que dans le cadre de l'écriture.

- la forme interne (la représentation) du signifié (l'« interprétant » selon Peirce) est normalement un processus interne, mental. Peirce admet en plus des interprétants abstraits qui sont eux-mêmes des signes - ce qui donne lieu à une itération infinie dans la position de l'interprétant.

- la forme externe (l'objet ou le processus qui sont externes au système cognitif). Il s'agit surtout du monde phénoménal accessible à la personne qui parle, écrit etc..., par exemple du contexte d'un locuteur, du monde dans lequel il agit, qu'il perçoit et dont il forme une représentation interne à l'aide de la mémoire et de l'imagination.

Graphe de la triade de Peirce

\section{Interprétant}

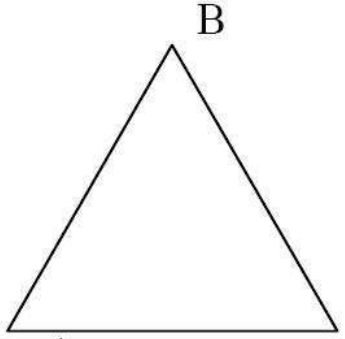

A

\section{$\mathrm{C}$}

\section{Representamen \\ Objet}

Pour donner quelques éléments de réponse à la question de savoir quel est le statut ontologique d'une entité qu'on appelle "signification", Widgen se propose de mettre en examen tout d'abord les trois postulats qu'il baptise: réaliste, peircien et génétique. Le postulat réaliste précise les trois types fondamentaux de réalité correspondant au phénomène de la signification: réalité scientifique, réalité phénoménale et réalité organique de l'homme. Il ne doit pourtant pas être pris au sens empirique, voire behavioriste. Le postulat de Peirce se donne pour but de le mettre à l'épreuve, non pas à titre d'évidence empirique, mais à titre de présupposition épistémologique. En fait ce qui fait l'originalité de la démarche de Widgen consiste à montrer d'une part que la triade de Peirce ainsi présentée constitue la condition de la théorisation possible des phénomènes sémantiques et d'autre part que cette condition était partiellement remplie dans l'histoire de la sémantique. Suivant les trois pôles A, B et C, parmi lequel on choisit le centre organisateur de tel ou tel modèle, on pourrait obtenir quatre types possibles de théorisation: structuralisme (Saussure), behaviorisme, cognitivisme, et platonisme. $\mathrm{Si}$ l'on met l'accent sur la dyade A-B, en laissant en arrière plan C, on obtient un paradigme structuraliste, de Saussure à Greimas en passant par Hjelmslev. Si l'on considère $C$ comme "stimulus", A comme "réaction" et B comme "boite noire", c'est le paradigme behavioriste qui se met en place, paradigme auquel appartient la tradition américaine, de C. S. Peirce à R. Carnap en passant par C. Morris. Dans le paradigme cognitiviste on choisit $\mathrm{B}$ comme centre organisateur d'un modèle; dans ce cas A n'est que la réalisation d'une représentation interne et $\mathrm{C}$ n'est qu'une conséquence externe d'une réalisation sensori- 
motrice dans divers actes cognitifs. Le platonisme se manifeste sous la forme d'un point de vue abstrait qui postule des structures logico-mathématiques sous-jacentes à $\mathrm{A}, \mathrm{B}$ et $\mathrm{C}$. Widgen vise la synthèse des hétérogènes paradigmatiques de la triade sémiotique, à partir du postulat génétique, c'est-à-dire dans le cadre d'une sémiotique morphodynamique, inaugurée par J. Petitot.

Mon objectif n'est pas la constitution d'une synthèse quelconque, mais la recherche de la façon dont la sémiotique et la sémantique s'articulent l'une à l'autre dans chaque paradigme. Il va sans dire qu'il y a plusieurs écoles et programmes de sémantique et de sémiotique, lesquelles ne communiquent pas et ne s'entendent pas, résistant ainsi à une quelconque catégorisation simpliste. Il ne sera donc pas question de les classer sous quelque rubrique que ce soit, mais de les caractériser épistémologiquement à partir de la triade de Peirce. Pour ce faire il nous faudrait suivre Widgen jusqu'au point où il reformule, pour rendre compte du postulat de Thom, les éléments constitutifs du triangle de Peirce en termes de catégories épistémologiques: linguistique (A), cognitif (B) et ontologique (C) (1994:169). J'aimerais les présenter à ma manière comme suit :

\section{Représentation cognitive \\ Forme du signifié \\ (cognitif)}

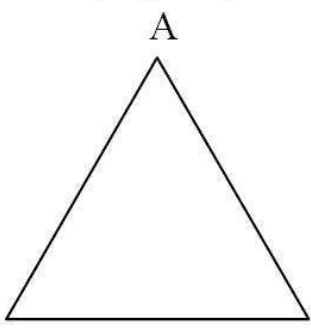

B $\mathrm{C}$

Structure linguistique

Forme du signe (linguistique/formel)
Ontologie

Forme du référent

(pragmatique/substantiel)

7 Ainsi reformulé, le triangle de Peirce ne désigne plus la structure du signe, mais celle de l'épistémologie. En fait «la structure ternaire de la signification », selon S. Auroux, « est avant tout la limite d'un champ culturel, celui de la sémiotique ». (1979: 66). Le triangle épistémique ainsi reformulé nous permettra de pénétrer dans le tréfonds de l'entrecroisement de la sémiotique et de la sémantique.

\section{Le sémantique dans la sémiotique}

Pour commencer, je pars de l'origine. De la double origine de la nouvelle science que F. de Saussure, et Ch. S. Peirce conçoivent presque à la même époque sous une dénomination légèrement différente : sémiologie pour le premier et sémiotique pour le second; origine de la confrontation indispensable pour la création d'une science. N'est-ce pas dans un contexte discursif qu'une science naissante réclame son droit de cité sur la scène scientifique ? Il faut saisir ce moment de création discursif : la nouvelle science des signes se crée en contrastant avec une autre science également novatrice, celle de la signification. Ce que M. Bréal voulait dire au nom de la sémantique ${ }^{3}$, ne serait-il pas un 
exemple de ce que Saussure voulait dire au nom de la sémiologie? N'est-ce pas ce que Lady Welby voulait démontrer au nom de la "signifique », qui serait finalement ce que Peirce voulait démontrer au nom de la sémiotique ? D’après G. Mounin, «la sémiologie n'est pas la sémantique » (1972: 8). Or cette thèse n'est vraie qu'en apparence. Elle n'empêche pas H. Aarsleff d'émettre l'hypothèse selon laquelle "Bréal's 'science des significations' was an example of Saussure's 'sémiologie' - a science that studies the life of signs within society " $(1982: 391)$. Il en serait de même du rapport entre la sémiotique de Peirce et la « signifique » que Lady Welby définit dans l'Encyclopaedia Britannica comme science de la signification (Peirce 1978 : 19). Lady Welby espère particulièrement « qu'on finira par se rendre compte que la sémiotique de Peirce et sa signifique sont étroitement liées l'une à l'autre comme il (Peirce) suggère qu'elle devraient l'être » (Peirce 1978 : 20). Ce serait une erreur de penser pour autant que la sémiotique et la signifique sont dotées d'un statut épistémologique identique. Même si elles sont étroitement liées l'une à l'autre, ce lien ne semble pas être égalitaire mais hiéarchique. Ainsi Peirce affirme que "La Signifique semblerait, de par son nom, être cette partie de la sémiotique qui cherche à déterminer la relation qu'entretiennent les signes avec leurs interprétants » (Peirce 1978 : 50). De même, si Bréal précède Saussure, cela n'empêche pourtant pas la sémiologie de celui-ci d'englober la sémantique de celui-là. Wunderli précise : «Die semantik ist ein Teilgebiet der Semiologie, allerdings in einem anderen Sinn : sie befasst sich nicht mit einem speziellen (privilegierten) System unter den semiologischen systemen, sondern mit einem besonderen Aspekt jedes einem solchen System angehörenden Zeichens : dem inhaltlichen ». (1981: 18).

9 La science des significations fait partie de la science des signes dans un sens particulier: pour Saussure au sens où le signifié est un élément constitutif du signe; pour Peirce au sens où «l'action du signe » produit «les effets signifiés propres des signes » (Peirce 1978 : 130). La question se pose d'emblée de savoir quelle sémantique pourrait contribuer à l'élucidation des éléments sémantiques contenus dans des systèmes signifiants. Rappelons que si la sémiologie saussurienne met l'accent sur la relation des signes, elle porte ses fruits les plus intéressants dans le structuralisme linguistique, voire dans la sémio-linguistique greimassienne; la sémiotique peircienne, quant à elle, attire l'attention sur l'action du signe et se développe sous l'optique du behaviorisme, reformulé par C. Morris (1955). Cela étant dit, la réponse à la question posée ci-dessus s'impose en quelque sorte : la sémantique structurale viendra en aide à la théorisation du concept de signe saussurien tandis que la pragmatique pourvoira à la théorisation du concept de sémiosis de Peirce. De toute façon l'important est que la sémiotique s'aide d'une sémantique.

Il est à noter du reste que la sémiotique de Greimas se constitue bien sous la forme d'une sémantique. Sémantique structurale, «le premier traité de sémiotique linguistique », selon $\mathrm{J}$-Cl. Coquet (1982:15), marqua ainsi une nouvelle ère de ce qu'on appelle communément la sémiotique textuelle ou littéraire. Coquet a bien saisi les trois moments de sa constitution: «A.J. Greimas voyait dans la lexicologie cette discipline qui serait peut-être en mesure de fournir l'outil théorique et méthodologique réclamé par les sciences de l'homme; en 1966, il substituait à la lexicologie la sémantique et, à partir de 1970, la sémiotique à la sémantique » (1982: 16). D'après lui, ce qui fait la spécificité de l'École de Paris par rapport aux autres courants de la sémiotique consiste précisément dans sa façon de définir ce qu'est la sémiotique. Il précise : «Une interview d'U. Eco portant sur son livre La structure absente commençait par cette définition : la sémiotique est la science des 
signes. Le Petit Robert dit de même, dans sa première édition: "Théorie générale des signes ». Pour l'École de Paris, la définition est autre. La sémiotique a pour projet d'établir une théorie générale des systèmes de signification " (1982: 5). L'accent est mis sur le terme de «signification ». Mais cette remarque de Coquet n'est vraie que partiellement. Par exemple, Thomas A. Sebeok, qui propose une approche biologique de la sémiotique, approche qui semble la plus éloignée des phénomènes de signification, souligne pourtant le fait que "The subject matter of semiotics, it is often credited, is the exchange of any messages whatsoever - in a word, communication. To this must at once be added that semiotics is also focally concerned with the study of signification " (1994:5). Si Eco définit la sémiotique comme science des signes, cette science a pour tâche de décrire tous les phénomènes de la signification. Ainsi dit-il dans son livre A Theory of Semiotics : «The aim of this book is to explore the theoretical possibility and the social function of a unified approach to every phenomenon of signification and/or communication. Such an approach should take the form of a general semiotic theory, able to explain every case of sign-function in terms of underlying systems of elements mutually correlated by one or more codes » (Eco 1979: 3). La différence entre Greimas et Eco ne résiderait pas dans leur façon de définir la sémiotique mais dans leur façon de définir ce qu'est la signification. Greimas se propose de définir la signification à partir de la perception: "C'est en connaissance de cause que nous proposons de considérer la perception comme le lieu non linguistique où se situe l'appréhension de la signification » (1966: 8). Il part, pour ainsi dire, de la Phénoménologie de la perception pour la description des phénomènes sémantiques. Il est à noter que cette position phénoménologique reste un dénominateur commun pour ses divers successeurs. (Coquet 1997, Petitot 1985 \& 1992, Rastier 1987). Par contre, Eco fait appel à la tradition de la logique, partant, pour la description du contenu sémantique, de la dichotomie dénotation/connotation, extension/intension, etc. Par voie de conséquence, on peut observer que, alors que Greimas reste en deçà de la sémantique structurale pour la description sémantique, Eco laisse une large part à la sémantique référentielle, par exemple celle de Katz et Fodor (cf. Eco 1979 : 96-98).

11 Issue de la sémio-linguistique de Greimas, Morphogenèse du sens (1985) de J. Petitot vise à la "naturalisation du sens", voire à la Physique du sens (1992: XIV). Qu'entend-il par "naturalisation du sens"? Ce serait une erreur de penser que le sens constitue une entité d'ordre naturel. Il reste toujours une entité d'ordre hautement culturel. Ce que J. Petitot entend par "naturalisation du sens ", c'est que celui-ci émerge de la nature. Et cette émergence du sens est soumise aux lois de la physique. Le sens ne fait pas l'objet de la description mais l'objet de l'explication. La distinction entre nature et culture n'est qu'une distinction de niveau. Ce qui est important, c'est que le niveau inférieur (nature) détermine l'émergence du niveau supérieur (culture). C'est précisément en ce sens que Petitot affirme : « les sciences humaines seront des sciences naturelles ou ne seront pas » (ibid.). En disant cela, il tente de battre en brèche le post-structuralisme qui, à ses yeux, se montre régressif « dans une sophistique et une dialectique anti-théorique » (ibid.) et de le remettre sur la bonne route sous une perspective génétique, celle de la morphodynamique proposée par R. Thom. Cette perspective thomienne se révèle être parfaitement coextensive au connexionisme dont se réclame la sémantique cognitive. $\mathrm{Si}$ la sémiotique morphodynamique de Petitot ne se réfère pas à la sémantique cognitive pour l'explication physique du sens, elle ne se développe qu'en contrastant avec elle. Par voie de conséquence, Petitot quitte la sémantique structurale pour la sémantique cognitive. Ainsi reconstituée, la sémiotique cognitive, d'après $\mathrm{P}$. Ouellet, a pour mission de jeter un pont entre la «perception sensorielle» et la "perception sémantique» 
(1994: 141). On dirait que le sémantique reste une problématique importante, voire une forme de savoir en sémiotique cognitive.

Par souci de clarté, j'aimerais établir un tableau capable de résumer clairement mon propos.

\begin{tabular}{|l|l|l|}
\hline Catégorie & Sémiotique & Sémantique \\
\hline \hline A : Linguistique & $\begin{array}{l}\text { Sémiotique formelle, } \\
\text { linguistique, textuelle, etc }\end{array}$ & Sémantique structurale \\
\hline B : Cognitif & $\begin{array}{l}\text { Sémiotique cognitive, } \\
\text { morphodynamique, etc }\end{array}$ & Sémantique cognitive \\
\hline C : Ontologique & Sémiotique substantielle, & Sémantique référentielle, \\
& biologique, etc & behavioriste \\
\hline
\end{tabular}

\section{Le sémiotique dans la sémantique}

13 Si la sémiotique se réfère explicitement ou implicitement à la sémantique déjà existante pour la description du contenu sémantique, la sémantique, de son côté, n'a pas à le faire. Cela ne nous empêche pourtant pas d'observer dans des manuels de sémantique l'existence d'une partie importante consacrée à la théorie des signes: A. Schaff (1960: 192-257), J. Lyons (1978: 82-100), P. Fabre (1978: 38-45), etc. Mais mon propos ne consistera pas à confirmer cette observation en énumérant la liste des références mais à chercher sous un angle épistémologique la raison pour laquelle la théorie de la signification présuppose, sinon la théorie, du moins la structure ternaire du signe. Dans la suite, je me contenterai, au lieu d'un détour historique, de donner un aperçu épistémologique.

D'après Rastier, l'univers de la sémantique, suivant la définition qu'on donne de ce qu'est la «signification", se divise en trois continents : référence, inférence et différence ${ }^{4}$. La sémantique référentielle réduit la signification d'un mot ou d'une phrase à la relation entre tel mot ou telle phrase et ce à quoi « il » ou « elle » renvoie. Le mot ne signifie pas, il désigne. La signification est ainsi réduite à la référence, et la sémantique s'épuise dans l'ontologie. Dans la triade de Peirce, c'est le pôle C qui prend relief, en faisant l'économie du pôle $B$. Il sert de critère d'évaluation pour la détermination d'une valeur de vérité. La relation sémiotique qui s'établit entre $\mathrm{A}$ et $\mathrm{C}$ s'explique là en termes purement et simplement symboliques. Il n'existe pas de signes mais seulement des symboles qui ne suivent que des règles syntaxiques. Par voie de conséquence, la sémantique perd son autonomie vis-à-vis de la syntaxe donnant des règles aux symboles. Un des enjeux principaux de la sémantique cognitive, d'après Petitot, consiste à démontrer qu'« il existe (pourtant) des contraintes sémantiques conditionnant la syntaxe» (1989: 77).

La sémantique inférentielle définit la signification comme intention d'un sujet parlant. Pour savoir ce que signifie tel ou tel mot ou telle ou telle phrase, il faut savoir ce que le sujet parlant entend par là. Le sens est ainsi chargé d'un contenu intentionnel. Pour déduire celui-ci à partir des expressions données, on recourt, comme c'était le cas de la 
sémantique de référence, à des calculs logiques. Un des enjeux les plus intéressants dans l'histoire de la sémantique $\mathrm{du} \mathrm{XX}^{\mathrm{e}}$ siècle consiste pourtant à intégrer la logique extensionnelle (référence) dans la logique intensionnelle ${ }^{5}$. Disons que la pragmatique complèterait la sémantique. Dans la structure ternaire du signe, c'est donc le pôle B qui joue un rôle intégrant. La valeur de vérité ne s'explique plus en termes extensionnels mais en termes intensionnels. La problématique de la vérité reste pourtant en vigueur. Par voie de conséquence, la sémantique de référence n'est pas disparue et n'est pas non plus réduite, mais soumise ou intégrée à la sémantique inférentielle. Et en ce sens on pourrait dire qu'elles demeurent toutes les deux en deçà de la catégorie ontologique.

Mais il y a un autre type de sémantique. Issue du structuralisme européen, la sémantique de différence se propose de fonder la signification, ni sur la référence, ni sur l'intention, mais sur la perception. "La perception sémantique", selon Rastier, saisit un écart différentiel entre des unités linguistiques (1991: 205). C'est précisément cet écart différentiel qui décide la signification de tel ou tel mot. Rastier précise: «le sens linguistique n'est pas (ou pas seulement) constitué par la référence à des choses, ou par l' inférence entre concepts, mais aussi et d'abord par la différence entre des unités linguistiques » (1991: 101). Bref, la signification provient de la différence. Si l'on se réfère à la triade de Peirce, c'est le pôle A qui est mis en relief dans la mesure où la perception sémantique ne porte que sur des unités linguistiques. Là, le pôle $C$ est laissé en arrière plan. Dans la sémantique de différence ainsi caractérisée, la relation sémiotique entre A et B s'explique en termes soit génératifs (Greimas 1966), soit interprétatifs (Rastier 1987). La sémantique ne dépend pas d'une compétence syntaxique mais de la sémiosis, « compétence sémiotique » selon Rastier. L'autonomie de la sémantique par rapport à la syntaxe reste donc intacte. Si la sémantique de différence peut être qualifiée de formelle, c'est parce qu'elle ne tient compte de l'objet que sémiotiquement constitué6. On dirait que le sémiotique constitue l'horizon épistémologique sur le fond duquel se détache toute forme de connaissance sémantique.

17 Rastier (1991) se demande dans lequel, parmi les trois continents ainsi identifiés, s'installe une nouvelle approche de la sémantique, à savoir, la sémantique cognitive. Il me semble qu'il compte sur le continent de la différence pour la réunification d'un empire du sens. La sémantique cognitive replace le concept de signe qui a disparu dans la grammaire générative de Chomsky, au centre de sa préoccupation, en renouant ainsi avec « the spirit of classic Saussurean diagram " (Langacker 1987: 11). La relation du signe ne sert pas simplement d'horizon épistémologique mais de terme métathéorique à la structuration d'une grammaire. La grammaire cognitive, d'après Langacker, ne connaît que trois composantes : sémantique, phonologique et symbolique (1994: 70). Ce serait une erreur de penser que cette dernière pourrait être comprise en termes syntaxiques, comme c'était précisément le cas de Chomsky. Langacker se propose de la considérer comme opération de symbolisation «where a correspondence is established between a semantic structure and a phonological structure » (1987: 73-74). Cette opération de symbolisation, autrement dit, la mise en relation sémiotique entre signifiant et signifié n'est pourtant pas arbitraire comme chez Saussure mais cognitivement motivée. On pourrait dire avec S. Badir que la grammaire cognitive ainsi programmée prend pour objet d'étude la " structure profonde de la pensée " ${ }^{7}$. C'est donc le pôle B qui, dans la triade de Peirce, joue un rôle organisateur; les pôles $\mathrm{A}$ et $\mathrm{C}$ ne sont que la réalisation, soit linguistique, soit motrice d'un schème interne, cognitif.

Par souci de clarté, j'aimerais résumer mon propos sous la forme du tableau qui suit : 


\begin{tabular}{|l|l|l|}
\hline Catégorie & Sémantique & Structure du signe \\
\hline A : Linguistique & Sémantique de différence & $\mathrm{A}^{*} \mathrm{~B}(\mathrm{C})$ \\
\hline B : Cognitif & Sémantique cognitive & $\mathrm{B}^{*} \mathrm{~A} C$ \\
\hline C : Ontologique & Sémantique de référence ou d'inférence & $\mathrm{C}^{*} \mathrm{~A}(\mathrm{~B})$ ou C B* A \\
\hline
\end{tabular}

(* : centre organisateur ; () : mise en arrière plan)

\section{Conclusion}

D’après G. Mounin, la sémiologie n'est pas la sémantique. La raison en est claire : alors que celle-ci se circonscrit à des significations linguistiques, celle-là étend son objet d'étude, au-delà des signification linguistiques, jusqu'à des phénomènes significatifs non linguistiques. Dans un autre sens, Rastier caractérise leur difference : « Semiotics (insofar as it is limited to signs) has produced theories of signification only, whereas semantics (when it treats texts, that is) is bound to produce theories of meaning ${ }^{8}$. Je pense que la relation entre sémiotique et sémantique est plus complexe et plus profonde qu'on ne le croit en général.

Dans la présente étude j'ai essayé de démontrer que la sémiotique et la sémantique s'emboîtent l'une dans l'autre historiquement et épistémologiquement. Pour ce faire je suis parti de l'hypothèse selon laquelle le sémantique est dans la sémiotique et que le sémiotique est dans la sémantique. J'ai tenté, en m'appuyant sur la triade de Peirce, reformulée par Widgen, de mettre à l'épreuve cette double hypothèse. En guise de conclusion, on peut observer d'une part que la sémiotique, pour la description du contenu sémantique, en appelle à une théorie de la signification et d'autre part que la sémantique, suivant la définition qu'elle donne de la signification, présuppose une différente forme de structure du signe. De quelque manière qu'on considère le signifié, soit comme une partie constitutive du signe (Saussure), soit comme un effet de l'action du signe (Peirce), la sémiotique, comme science des signes, ne pourra s'en passer, c'est-à-dire qu'elle s'aidera d'une sémantique pour en donner une description théorique. La sémantique, de son côté, doit s'interroger sur la question de savoir quel est le statut ontologique, épistémologique et méthodologique de ce qu'on appelle signification. Pour formuler une réponse à cette question, elle est tenue de présupposer inévitablement une structure du signe. Bref, si la sémiotique fait valoir une théorie de la signification pour la description sémantique, la sémantique présuppose une structure du signe pour la théorisation du sens. 


\section{BIBLIOGRAPHIE}

Aarsleff H. 1982 : From Locke to Saussure, London, Athlone.

Auroux S. 1979 : La sémiotique des encyclopédistes, Paris, Payot.

Badir S. : «Immanence et Cognitivisme. Un débat épistémologique autour de la sémantique cognitive », www.revue-texto.net/inedits/Badir/Badir.html.

Bréal M. 1897 : Essai de sémantique, Paris, Hachette.

Coquet J-Cl. et al. 1982 : Sémiotique. L'École de Paris, Paris, Classiques Hachette.

— 1997 : La quête du sens, Paris, PUF (= Formes sémiotiques).

Eco U. 1979 : A Theory of Semiotics, A Midland Book.

Fabre P. 1978 : La sémantique, Paris, Nathan.

Greimas A. J. 1966 : Sémantique structurale, Paris, Larousse.

Langacker R. W. 1987 : Foundations of Cognitive Grammar, vol. 1, Stanford University Press.

- 1994 : «Structural Syntax : The view from Cognitive Grammar », Sémiotiques, 6-7.

Lyons J. 1978 : Éléments de sémantique, Paris, Larousse.

Morris Ch. 1946 : Signs Language and Behavior, New York, George Braziller Inc.

Mounin G. 1972 : Clefs pour la sémantique, Paris, Seghers.

Ouellet P. 1994 : «La sémiotique cognitive : les sciences de l'esprit entre la nature et la culture », Sémiotiques, 6-7.

Peirce Ch. S. 1978 : Écrits sur le signe, rassemblés, traduits et commentés par G. Deledalle, Paris, Le Seuil.

Petitot J. 1985 : Morphogenèse du Sens, Paris, PUF.

- 1989 : « Hypothèse localiste, modèles morphodynamiques, et théories cognitives : Remarques sur une note de $1975 »$, Semiotica 77-1/3.

- 1992 : Physique du Sens, Paris, Éditions du C.N.R.S.

Rastier Fr. 1987 : Sémiotique interprétative, Paris, PUF.

- 1991 : Sémantique et recherches cognitives, Paris, PUF.

- : « On Signs and Text », www.chass.utoronto.ca/french/as-sa/ASSA-No5/FR1.htm.

Saussure F. de 1916 : Cours de linguistique générale, Paris, Payot.

Schaff A. 1960 : Introduction à la sémantique, Paris, Anthropos.

Sebeok T.A. 1994 : An introduction to Semiotics, University of Toronto Press.

Tamba-Mecz I. 1988 : La sémantique, Paris, PUF.

Widgen W. 1994 : « Les fondements sémiotiques et philosophiques d'une grammaire morphodynamique », Sémiotiques, 6-7. 
Wunderli P. 1981 : «Zur Textkritik von Sémantique und Sémiologie », in Saussure-Studien, Tübingen, Gunter Narr Verlag.

\section{NOTES}

1. F. Rastier, www.chass.utoronto.ca/french/as-sa/ASSA-No/FR1.htm., p. 321.

2. Il s'agit du beau titre d'un ouvrage de J-Cl. Coquet (1997).

3. M. Bréal (1897) qu'on reconnaît comme fondateur de ce qu'on appelle la sémantique donne de celle-ci la définition suivante dans laquelle Irène Tamba-Mecz voit «l'intuition dont est sortie toute la sémantique » $(1988: 4):$ «L'étude où nous invitons le lecteur à nous suivre est d'espèce si nouvelle qu'elle n'a même pas encore reçu de nom. En effet, c'est sur le corps et sur la forme des mots que la plupart des linguistes ont exercé leur sagacité : les lois qui président à la transformation des sens, au choix d'expressions nouvelles, à la naissance et à la mort des locutions, ont été laissées dans l'ombre ou n'ont été indiquées qu'en passant. Comme cette étude, aussi bien que la phonétique et la morphologie, mérite d'avoir son nom, nous l'appellerons la sémantique, c'est-à-dire la science des significations » (ibid., p.11-12)

4. F. Rastier $1991: 82-88,97-107$.

5. Il ne faut pas confondre la sémantique d'inférence avec la logique intensionnelle. Si la première relève de l'ordre de la parole, c'est-à-dire, de la pragmatique, la deuxième reste attachée à la description formelle du contenu sémantique, ignorant ainsi des variétés situationnelles. Il est à noter toutefois que la sémantique d'inférence fait appel à la logique intensionnelle (non à la logique extensionnelle) pour la description du sens en contexte.

6. Rastier, $1987: 19$.

7. Badir, www.revue-texto.net/inedits/Badir/Badir.html. p. 15.

8. F. Rastier : www.chass.utoronto.ca/french/as-sa/ASSA-No5/FR1.htm. p. 321.

\section{RÉSUMÉS}

Comment définir la relation entre sémiotique et sémantique ? On dit que, alors que la sémantique concerne la signification linguistique, la sémiotique étend son objet d'étude jusqu'à des phénomènes significatifs non linguistiques. Cette position simpliste nous empêche de nous confronter à la complexité de la question. La relation entre sémiotique et sémantique, à mes yeux, est plus complexe et plus profonde qu'on ne le croit en général. Pour donner quelques éléments de réponse à cette question, je suis parti de l'hypothèse suivante : le sémantique est dans la sémiotique et le sémiotique est dans la sémantique. Et j'en dégage la conclusion suivante : - si le signifié est un élément constitutif du signe et que la sémiotique se définit comme science des signes, cette science a besoin d'une théorie sémantique pour la description sémantique.

- comme science de la signification, la sémantique doit s'interroger sur le statut épistémologique, ontologique et méthologique de ce qu'on appelle signification. Pour formuler une réponse à cette question incontournable, elle est tenue de présupposer une structure du signe.

How to define the relation between semiotics and semantics? One can say that semantics is only concerned with the linguistic meaning, while semiotics extends its research fields to the nonlinguistic phenomena. It seems to me that this simple fact hides the complexity of the question. 
In fact their relation is more complex and more profound. To throw some light on the question, I began with the hypothesis that the semantic is in semiotics and the semiotic is in semantics. And I draw the conclusion as these:

- if the signified is a constitutive part of sign and if semiotics is defined as science of signs, this science needs necessarily semantic theory.

- as science of significations, semantics asks itself which ontological, epistemological, and methodological status should be accorded to what people call signification. In order to give an adequate answer to this question, it presupposes necessarily sign structure.

\section{AUTEUR}

\section{YONG-HO CHOI}

Université Hankuk des Etudes Etrangères, Département de Français, Corée du sud, e-mail : desaussure@hanmail.net 